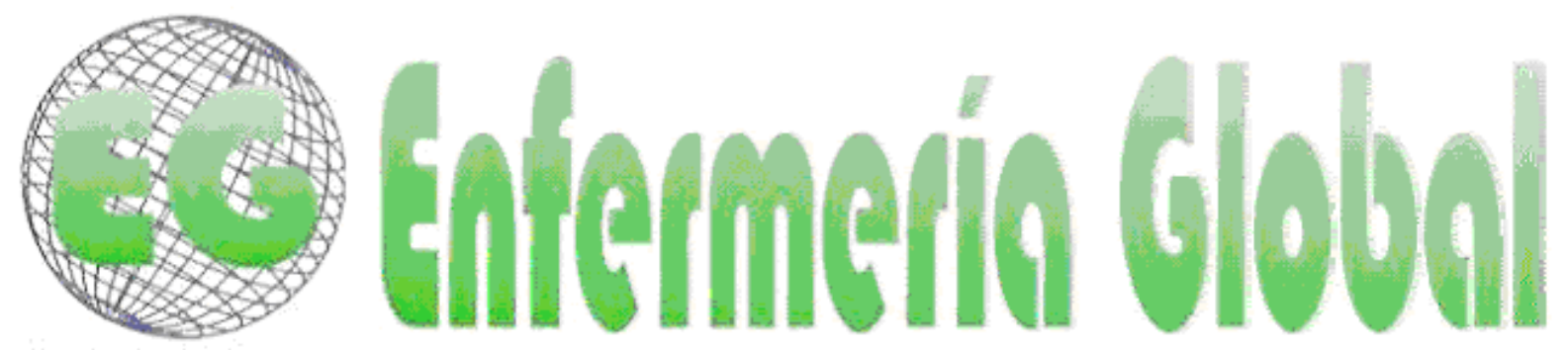

\title{
Teoría de los cuidados de Swanson y sus fundamentos, una teoría de mediano rango para la enfermería profesional en Chile.
}

Swanson's care theory and her fundamental ideas, a mid-range theory for professional nursing in Chile.

\section{${ }^{*}$ Rodríguez Campo, VA., "*Valenzuela Suazo, S.}

\author{
*Magíster en Enfermería. Candidata a Doctora en Enfermería. Profesora de Biología- E-mail: \\ varirodriguez@udec.cl **Doctora en Enfermería. Profesora Titular Departamento de Enfermería. \\ Universidad de Concepción. Chile.
}

Palabras clave: Teoría; Gestión del Cuidado; Enfermería.

Keywords: Theory; Care Management; Nursing.

\section{RESUMEN}

La estructura del conocimiento es fundamentado en teorías que permiten el actuar del profesional de enfermería.

Objetivo: Analizar la teoría de Los Cuidados de Kristen Swanson para sustentar los cuidados profesionales de enfermería en Chile.

Metodología: Se realizó revisión de bibliografía en bases de datos Scielo, Lilacs, Pubmed.

Resultados: Las teorías permiten la generación del conocimiento, perfeccionan las prácticas y fundamentan el cuidado profesional en enfermería.

Conclusión: El rol gestor del cuidado que se atribuye a los profesionales de enfermería en Chile puede y debe ser respaldado por conocimientos teóricos que aseguren la calidad de los cuidados hacia el usuario.

\section{ABSTRACT}

The structure of knowledge is based on theories that allow the work of a Nursing Professional.

Objective: To analyze Kristen Swanson's Care Theory to support Nursing's professional health care in Chile.

Methodology: A revision of the bibliography was performed on the databases scielo, lilacs and pubmed. 
Results: The theories encourage the creation of knowledge, they perfect the health care practices and provide the fundamentals for Nursing's professional care.

Conclusion: The Care Management Role given to the nursing professionals in Chile can and should be backed up by theoretical knowledge that will insure quality care towards the patient.

\section{INTRODUCCIÓN}

La disciplina de enfermería ha ido evolucionando con los acontecimientos y con las diversas corrientes del pensamiento a través de la historia. El cuidado en enfermería comienza desde los inicios de la vida y se han destacado cuatro etapas en este proceso hasta convertirla en profesión: la etapa doméstica, vocacional, técnica y profesional ${ }^{(1)}$ Aproximadamente en el siglo XIX Nightingale define al cuidado como "un arte y una ciencia que exige una formación formal y el papel de la enfermera es poner al sujeto en las mejores condiciones para que la naturaleza actúe sobre él" y da comienzo a la profesionalización de enfermería ${ }^{(2)}$.

A partir del año 1997, en Chile, los servicios profesionales de la enfermera comprenden la Gestión del Cuidado en lo relativo a promoción, manutención y restauración de la salud, la prevención de enfermedades o lesiones, y la ejecución de acciones derivadas del diagnóstico y tratamiento médico y el deber de velar por la mejor administración de los recursos de asistencia para el paciente ${ }^{(3)}$. Este reconocimiento significa que el cuidado es el aporte especifico de la enfermera en la atención de salud y, además, un ámbito determinado de esta atención en salud ha sido reservado para las enfermeras, con el objeto de otorgar certeza jurídica respecto a su campo de acción; de esta manera se garantiza el derecho a la protección de la salud del usuario ${ }^{(4)}$

La Gestión del Cuidado de enfermería será entendida como el ejercicio profesional de la enfermera sustentada en su disciplina: la ciencia del cuidar. Esta se define como la aplicación de un juicio profesional en la planificación, organización, motivación y control de la provisión de cuidados, oportunos, seguros, integrales, que aseguren la continuidad de la atención y se sustenten en las políticas y lineamientos estratégicos de la institución Por lo tanto, su fin último es ofrecer a los usuarios los mejores resultados posibles en la práctica diaria, acorde con la información científica disponible que haya demostrado su capacidad para cambiar de forma favorable el curso clínico de la enfermedad y que considere la mejor administración de los recursos, los menores inconvenientes y costos para el usuario y para la sociedad en su conjunto ${ }^{(5)}$.

El objetivo de la presente revisión bibliográfica es el análisis de la teoría de los Cuidados, de Kristen Swanson para sustentar los cuidados profesionales de enfermería, tanto a nivel asistencial como académico.

\section{TEORÍA DE LOS CUIDADOS DE KRISTEN SWANSON}

Las teorías buscan describir, explicar, predecir y controlar los fenómenos de tal manera, de adquirir conocimientos para perfeccionar las prácticas. Es así, que las teorías han permitido a enfermería mejorar su capacidad, profesional y disciplinar, a través del conocimiento, pues los métodos aplicados de forma sistemática tienen mayor probabilidad de éxito ${ }^{(2 ; 6)}$. Las teorías estimulan el razonamiento que se aplican a suposiciones y determinan los objetivos de la práctica, la educación y la 
investigación. Son las teorías quienes permiten la generación del conocimiento (lo único que puede explicar el mundo de enfermería), y de esta manera avanzar en la conformación de un cuerpo propio de conocimientos disciplinares. Los modelos conceptuales reflejan la riqueza del pensamiento enfermero que está continuamente en evolución ${ }^{(7)}$. El conocimiento abstracto es el que permite que los hallazgos científicos derivados de investigación se desarrollen en teorías que, guiadas por un soporte filosófico, puedan sustentar la práctica. Se puede asegurar que la teoría es uno de los componentes de la estructura del conocimiento que nos permite dar sentido al mundo empírico y, por tanto, entender de una manera más coherente y controlada nuestra práctica ${ }^{(8)}$. Las enfermeras ya no pueden resumir las experiencias en salud de las personas en base a diagnósticos médicos. Los modelos conceptuales y las teorías existen para lanzar desafíos a la práctica. El conocimiento adquirido a través de la teoría estimula el pensamiento creativo, guía la enseñanza y la investigación ${ }^{(7)}$

Enfermería como disciplina, está compuesta por varios componentes entre los que destacan, perspectiva, dominio, definiciones y conceptos existentes y aceptados por la enfermería y patrones de conocimientos de la disciplina ${ }^{(9)}$ por lo que depende de la existencia y generación de un conocimiento teórico que sustente todas las áreas. La aplicación de un Modelo Conceptual o Teoría de Enfermería en la atención, garantiza que todas las enfermeras compartan igual concepción del metaparadigma e igual lenguaje en la orientación de los cuidados ${ }^{(10)}$. De mayor a menor nivel de abstracción los modelos y teorías de enfermería se estructuran desde lo más filosófico a lo más explicativo en metaparadigmas, filosofías, modelos conceptuales, grandes teorías, teorías y teorías de rango medio.

El metaparadigma es el nivel de conocimientos más abstracto de todos. Describe los principales conceptos que hacen referencia al tema principal, así como a la finalidad de una disciplina. Los principales conceptos del metaparadigma enfermero son el de persona, entorno, salud y enfermería (11). Las grandes teorías son aquellas que cubren grandes áreas de preocupación dentro de una disciplina. Son construcciones sistemáticas sobre la naturaleza de la enfermería, la misión de la enfermería y el cuidado de enfermería. Adquieren un papel formativo y de socialización, sin embargo, la macroteoría comienza a decrecer a medida que generan conocimiento, dando paso a las teorías de rango medio. Estas últimas, son mucho mas limitadas en amplitud, menos abstractas, y reflejan de mejor manera los componentes de la práctica ${ }^{\left({ }^{(9)}\right.}$. Se describen como comprobables e intermedias en su ámbito, adecuadas en fundamento empírico: ni muy amplias ni muy estrechas, circunscritas y sustantivamente específicas. Se caracterizan por explicar y predecir un fenómeno (concepto), el cual debe ser lo suficientemente abstracto para ser aplicado en diferentes lugares y con diferentes problemas, es decir, deben ser fenómenos observables, donde se implique la habilidad de definir y capturar un aspecto de la experiencia con instrumentación válida y confiable, que permita predecir resultados que puedan ocurrir y explicar la relación entre el diagnóstico, la intervención y el resultado ${ }^{(11)}$. Meleis in Durán de Villalobos ${ }^{(9)}$ planteó que las grandes teorías permiten generar parámetros generalizados de la práctica, mientras que las teorías de rango medio generan guías más específicas de la misma.

Kristen Swanson plantea en el año 1991 la "Teoría de los Cuidados", la autora concibe los cuidados como una forma educativa de relacionarse con un ser apreciado hacia el que se siente un compromiso y una responsabilidad personal. Propone cinco procesos básicos (Conocimientos, estar con, hacer por, posibilitar y mantener las 
creencias). Dichos conceptos permiten reflexionar acerca de la cosmovisión del cuidado enfermero, donde convergen las dimensiones históricas, antropológicas y filosóficas de la ciencia de enfermería ${ }^{(12)}$. Los conocimientos se refieren a las capacidades de la persona para dar cuidados, el segundo las preocupaciones y compromisos individuales que llevan a las acciones de los cuidados, el tercero a las condiciones: enfermera, cliente, organización, que aumentan o reducen la probabilidad de suministrar cuidados, el cuarto a las acciones de los cuidados dados y por último hace referencia a las consecuencias de los resultados intencionales y no intencionales de los cuidados para el cliente y para el profesional ${ }^{(13)}$. La teoría apoya la reivindicación de que los cuidados son un fenómeno central de enfermería, pero no es necesariamente la práctica de enfermería ${ }^{(14)}$ Sanabria menciona que los cuidados de enfermería no son empíricos ni producto de una necesidad ocasional, sino que se fundamentan en un conocimiento teórico que facilita la interpretación y el análisis del cuidado que se brinda, se basan en una deducción lógica y en la explicación científica. ${ }^{(15)}$

\section{TEORÍA DE LOS CUIDADOS Y GESTIÓN DEL CUIDADO EN ENFERMERÍA}

En muchas ocasiones, "en la práctica de enfermería se observa que el cuidado es identificado como un quehacer monótono, lineal y sin sentido, dentro de la racionalidad técnica y el enfoque biomédico, entendido como una ayuda 0 un complemento en las ciencias de la salud, situación que propicia la negación de la enfermería como ciencia y como arte" $\left({ }^{16}\right)$. En Chile, autoras determinaron que aún en las escuelas de enfermería de ciertas universidades se mantiene el modelo biomédico en experiencias hospitalarias, por lo que se hace menor el rol del ejercicio autónomo de enfermería como gestor del cuidado ${ }^{(17)}$. En virtud de su contexto histórico, la enfermería tiene su cuerpo de conocimientos relativamente nuevo y en plena construcción, el cual ha desarrollado a través de las investigaciones. Cada trabajo producido avala la construcción y consolidación de su corpus teórico ${ }^{(18)}$ añadiendo nuevas modalidades de cuidar, aportando mejorías para la calidad de la atención, enseñanza e investigación con el consecuente mejoramiento de la profesión y avanzar en la construcción del conocimiento en enfermería.

La Enfermería tiene como fundamento una teoría que guía su práctica. El enfermero/a emplea las fuerzas de esa teoría en sus actividades asistenciales diariamente, aunque quizás no lo reconozca como tal. La práctica científica de la Enfermería requiere la adopción de una teoría que dé significado a la realidad donde se ejecutan los cuidados, y un método sistemático para determinar, organizar, realizar y evaluar las intervenciones de Enfermería ${ }^{(19)}$. Muchas teoristas han fundamentado el quehacer de enfermería en el cuidado, como Dorotea Orem y su teoría de déficit de Autocuidado $\left({ }^{20}\right)$, Jean Watson y su teoría Filosofía y Ciencia del Cuidado Transpersonal ${ }^{(21)}$, Madeleine Leininger y su teoría Cuidados Culturales: Teoría de la diversidad y universalidad ${ }^{(22)}$ y Kristen Swanson que nos propone la Teoría de los Cuidados.

El profesional de enfermería en Chile debe organizar, supervisar, evaluar y promover la calidad de cuidados de enfermería, con la finalidad de brindar atención segura, oportuna, continua e intercultural de acuerdo a las políticas y normas del ministerio de salud chileno. Debe participar en los lineamientos estratégicos relacionados con la dotación de recursos humanos, materiales, físicos y financieros del cuidado de enfermería, incluyendo la administración presupuestaria asignada y la implementación de nuevas herramientas tecnológicas que faciliten el control de la gestión. Debe 
promover los principios éticos y legales que guían el ejercicio profesional, tanto en su rol independiente como parte de un equipo multidisciplinario, en donde se promueva la comunicación eficaz entre las diferentes dependencias institucionales. La enfermera/o debe promover un liderazgo efectivo en la gestión de los equipos de trabajo de su dependencia, para ello debe proponer programas de evaluación y mejoramiento continuo del cuidado de enfermería. Se espera también que exista una integración docente asistencial para así incentivar la investigación en el ámbito de la gestión del cuidado ${ }^{(5)}$

Smith y Durán de Villalobos in Campos ${ }^{(23)}$, postulan que "el foco central del saber y hacer de la enfermería, es el cuidado, no existe otra profesión que esté tan comprometida con el proceso de cuidar, las acciones de cuidado, y con una relación interpersonal de cuidado". El cuidado es su concepto nuclear, define e identifica la disciplina, da cuenta del compromiso social de la profesión y acota el área de responsabilidad. Es por ello la importancia de una formación sistemática, rigurosa y especializada en los ámbitos del saber, hacer y ser que asegure a la población un cuidado de la más alta calidad ${ }^{(23)}$ en donde los nuevos profesionales enfermeras/os, que asuman la responsabilidad del cuidado como bien profesional que enriquezca a la disciplina, es por ello la importancia de quienes son los encargados de enseñar este proceso de cuidar. En Chile son las Universidades, públicas y privadas quienes otorgan la preparación de los futuros profesionales de enfermería ${ }^{(24)}$, en su actividad formadora y de investigación, incorpora contenidos éticos propios para cada profesión, de forma que el futuro profesional, además de lograr ser un experto en su materia, se encuentra en condiciones de actuar con base a criterios éticos. La formación universitaria no se reduce a incrementar el conocimiento especializado sino que incorpora aprendizajes que permitan el desarrollo ético de la persona, tanto en su dimensión individual como social ${ }^{(24 ; 25)}$.

Las enfermeras al prestar cuidados no deben perder la vista del individuo como un ser holístico, por lo que no sólo deben prestar atención a lo biológico sino que saber escuchar con atención, esto constituye la fase inicial del dialogo y permite la comunicación con la persona ${ }^{(26)}$, es más la enfermera/o debe ser capaz de respetar los derechos de los pacientes durante su cuidado, incluye la confidencialidad, derecho a la información, compañía, ayuda espiritual y participar del sistema de salud chileno(27). Swanson en Wojnar ${ }^{(13)}$ plantea que "independiente de los años de experiencia de una enfermera, los cuidados se dan como un conjunto de procesos secuenciales (subconceptos) creados por la propia actitud filosófica de la enfermera (mantener las creencias), la comprensión (conocimientos), los mensajes verbales y no verbales transmitidos al cliente (estar con) las acciones terapéuticas (hacer por y posibilitar) y las consecuencias de los cuidados (desenlace deseado por el cliente) Por lo tanto los cuidados se basan en el mantenimiento de una creencia básica de los seres humanos, apoyada por el conocimiento de la realidad del cliente, expresada por estar física y emocionalmente presente y representada por hacer y posibilitar al cliente".

Ceballos, (28) postula que se debe trabajar en la formación de futuras/os enfermeras/os, para que éstos comprendan la importancia de los cuidados humanizados para el paciente, esta imagen debe caracterizarse por intervenciones que demuestren calidad científica y humanización del cuidado. Los docentes deben ser modelos de formación para sus alumnos, por lo que primeramente son ellos los que deben cultivar estas competencias ${ }^{(29)}$ El cuidado, si bien es concebido como una actividad práctica, necesita de la actividad intelectual y de una masa crítica de 
investigadores e ideólogos que orienten las acciones, situación que debe fundamentarse en la investigación ${ }^{(30)}$. Hoy en día un gran número de enfermeras están comprometidas con una práctica profesional al lado de personas, familias o comunidades, y son responsables de ofrecer cuidados específicos basados en las necesidades que viven las personas ante diversas experiencias de salud ${ }^{(31)}$

\section{CONSIDERACIONES FINALES}

Sin duda como profesionales de enfermería la mejor forma de sustentar nuestros conocimientos es a través de teorías que intentan explicar y definir diversos fenómenos relacionados con la disciplina. El rol gestor del cuidado que se atribuye a los profesionales de enfermería en Chile puede y debe ser respaldado por conocimientos teóricos que aseguren la calidad de los cuidados hacia el usuario, tomando las mejores decisiones fundamentadas en teorías, de manera tal que sea respetado como profesional con conductas responsables de cuidado frente a la sociedad.

\section{REFERENCIAS}

1 García C, Martínez M. Historia de la enfermería. Evolución histórica del Cuidado Enfermero. Madrid, España: 2001.

2 Marriner A, Raile M. Modelos y teorías en Enfermería. Harcourt ed. Madrid, españa: 1999.

3 Código Sanitario. D.F.L 725/67, 725/67, Ministerio de Salud de Chile, (1968).

4 Milos P, Borquez B, Larraín A. "La Gestión del Cuidado en la Legislación Chilena: interpretación y Análisis". Cienc.enferm. 2010;16(1):17-29.

5 Aprueba Norma General Administrativa n 19, "Gestión del cuidado de Enfermería para la atención Cerrada", Exenta 1127, Subsecretaria de Redes Asistenciales, (2007).

6 Vargas M. Algunos fundamentos teóricos para el cuidado de enfermería. Rev Mexicana de Enfermería Cardiológica 2007;15(1):28-30.

7 Kérouac S, Pepin J, Ducharme F, Duquette A, Major F. El pensamiento enfermero. 1 ed. Barcelona, España: 2002.

8 Durán de Villalobos M. Teoría de Enfermería ¿un camino de herradura? Aquichán 2007;7(2):161-73.

9 Durán de Villalobos M. Marco Epistemológico de la Enfermería. Aquichán 2002;2(2):7-18.

10 Varez S, López M, Santos S, Abril D. Perspectiva sobre modelos y teorías de enfermería en el ámbito de nefrología. Rev Soc Esp Enferm Nefrol 2008;11(3):178-83.

11 Marriner A, Raile M. Modelos y teorías en enfermería. Sexta ed. Madrid.España: 2007.

12 Báez-Hernández F, Nava-Navarro V, Ramos-Cedeño L, Medina.López O. El significado de cuidado en la práctica profesional de enfermería. Rev Aquichan 2009;9(2).

13 Wojnar D. Kristen Swanson: Teoría de los Cuidados. In: Elsevier, editor. Modelos y teorías en enfermería. Sexta ed. Madrid.España: 2007. p. 766-77.

14 Potter P, Perry A. Fundamentos de Enfermería. 5 ed. Madrid.España: 2001.

15 Sanabria L, Otero M, Urbina O. Los paradigmas como base del pensamiento actual en la profesión de enfermería. Rev Cubana Educ Med Super 2002;16(4).

16 Medina J. La pedagogía del cuidado: saberes y prácticas en la formación universitaria en enfermería. 1 ed. Madrid.España: 1999. 
17 Sanhueza O, Jara P. Incorporando la Gestión del Cuidado en la Formación de Pregrado. Rev. Enfermería 2005;21(129):15-9.

18 Erdmann A, Leite J, Mendes I, Trevizan M, Dantas C. Analisis de investigaciones brasileñas enfocadas en el cuidado de enfermería, años 2001-2003. Rev Cienc. enferm. 2005;11(2):35-46.

19 León C. Enfermería ciencia y arte. Rev Cubana Enfermer. 2006; 22(4)

20 Taylor S, Compton A, Donohue J, Emerson S, Gashti N, Marriner A, et al. Dorothea E. Orem: teoría del deficit de autocuidado. In: Harcourt, editor. Modelos y teorías en enfermería. 4 ed. Madrid, España: 1999. p. 175-94.

21 Patton T, Barnhart D, Bennett P, Di Porter B, Sloan R. Jean Watson: filosofía y ciencia de la asistencia. In: Harcourt, editor. Modelos y teorias en Enfermería. 4 ed. Madrid, España: 1999. p. 142-56.

22 Welch A, Alexander J, Beagle C, Butler P, Dougherty D, Andrews K, et al. Madeleine Leininger: Cuidados culturales: teoría de la diversidad y universalidad. In: Harcourt, editor. Modelos y teorías en Enfermería. 4 ed. Madrid, España: 1999. p. 439-62.

23Campos C. Enfermería: Profesión y disciplina del cuidado*. Revista Enfermería 2008;1-4.

24 Milos $\mathrm{P}$, Larrain A, Simonetti M. Del porqué de la formación exclusiva universitaria de las enfermeras. 2009. Hallada en http/www.colegiodeenfermeras.cl

25 Milos P, Larrain A, Simonetti M. Categorización de Servicios de Enfermería.Propuesta para asegurar una atención de calidad en tiempos de escasez de enfermeras. Cienc. enferm. 2009;15(1):17-24.

26Rocha N, Costa I, Da Silva L, Monteiro A, De Melo A. La creatividad y cuidado crítico: contribuciones de la educación tanto de Paulo Freire para la enfermería . Cienc. enferm 2009;15(2):35-40.

27 Mirada en Profundidad. Derechos y deberes de los pacientes: habrá mayor claridad. El Mercurio . 2007.

28 Ceballos $\mathrm{P}$. Desde los ámbitos de enfermería, analizando el cuidado humanizado. Cienc. enferm. 2010;16(1):31-5.

29 Davies B, Hughes A. Clarification of advanced nursing practice: characteristics and competencies. Clinical Nurse Specialist 2002;16(3):147-52.

30 Rivas E, Osorio X. Estado de la investigacion en enfermería IX región de la Araucania, Temuco, Chile 2002a. Investigación / Research 2005;23(2):82-93.

31 Zarate R. La Gestión del Cuidado de Enfermería . Index Enferm 2004;13(44).

ISSN 1695-6141

(C) COPYRIGHT Servicio de Publicaciones - Universidad de Murcia 